\title{
Aplicaciones móviles aplicados desde estudios universitarios al Inbound marketing para la comercialización de productos agropecuarios
}

\author{
Mobile applications applied from university studies to \\ Inbound marketing for the marketing of agricultural \\ products
}

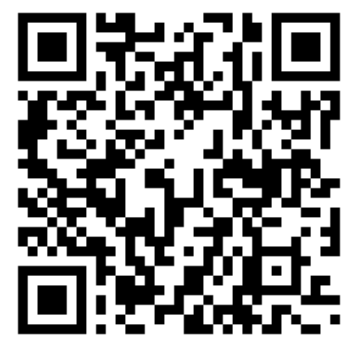

Francisco Javier González González ${ }^{* *}$ José Paúl Ronquillo Carrión ${ }^{* * *}$ Harry Alexander Vite Cevallos ${ }^{* * * *}$ Héctor Carvajal Romero*****

\section{Resumen}

Las empresas en la actualidad son cada día más dependientes de la tecnología, tanto para la producción como para la comercialización de sus productos y servicios, entre estas tecnologías se encuentra la interacción entre cliente y empresa, es por ello que se ha planteado

\footnotetext{
* Artículo original derivado del Proyecto "Aplicaciones moviles en el proceso de enseñanza" Financiado por la Universidad Técnica de Machala entre agosto 2018 y mayo 2019

** Docente Universitaria. Universidad Técnica de Machala. Machala, Ecuador. Email: fjgonzalez_est@utmachala.edu.ec. ORCID: http://orcid.org/0000-0002-49564312 .

*** Docente Universitaria. Universidad Técnica de Machala. Machala, Ecuador. Email: jpronquillo_est@utmachala.edu. ORCID: http://orcid.org/0000-0002-97477547

**** Docente Universitaria. Universidad Técnica de Machala. Machala, Ecuador. Email: hvite@utmachala.edu.ec. ORCID: http://orcid.org/0000-0003-2056-7111.

***** Docente Universitaria. Universidad Técnica de Machala. Machala, Ecuador. Email: fjgonzalez_est@utmachala.edu.ec. ORCID: http://orcid.org/0000-0001-63036295.
}

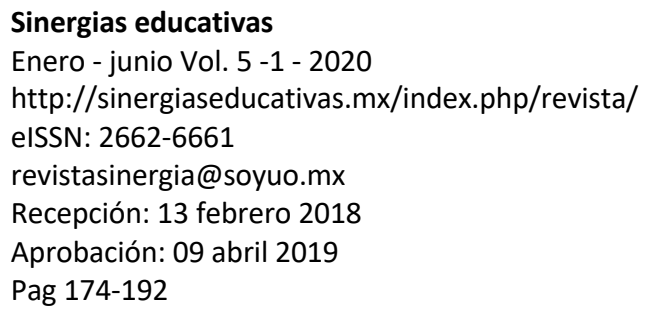

Disponible en

http://www.redalyc.org/articulo.oa?id=57356143200 13

Atribución/Reconocimiento-NoComercialCompartirlgual 4.0 Licencia Pública Internacional CC BY-NC-SA 4.0 
como objetivo elaborar un análisis bibliométrico acerca de las tendencias del uso de Inbound marketing en la comercialización de productos agropecuarios, para ello se procedió a investigar los diferentes artículos científicos publicados en relación al tema en los últimos cuatro años, para ello se utilizó las diferentes bases de datos, entre ellas Web of Science y Scopus y Taylor \& Francis, los términos que se utilizaron fueron "Marketing digital e Inbound marketing", además de ello, se analizó el tema específico Marketing digital en la comercialización de productos agrícolas, tanto en inglés como en español, al momento de escoger los artículos relacionados con la temática se utilizaron las técnicas del análisis síntesis, inductivo y deductivo, luego hacer una revisión de 50 artículos alusivos al tema se pudo concluir que la información existente al respecto es escasa, el sector alimentario ha sido muy poco explorado en este ámbito, incluso en muchas de las investigaciones revisadas todavía se confunde términos como Inbound marketing, marketing de contenidos y marketing digital, razón por la cual hay mucho que investigar al respecto, no solo como herramienta de comercialización para el sector agropecuario, sino también de manera general para los diferentes sectores económicos.

Palabras clave: comercialización, tecnologías, interacción, marketing digital.

\begin{abstract}
Companies today are increasingly dependent on technology, both for the production and for the marketing of their products and services, among these technologies is the interaction between client and company, which is why it has been proposed as an objective to make a bibliographic review about the use of Inbound marketing in the commercialization of agricultural products, for this we proceeded to investigate the different scientific articles published in relation to the subject in the last four years, for this the different databases were used, among them Web of Science and Scopus and Taylor \& Francis, the terms used were "Digital Marketing and Inbound marketing", in addition to this, the specific topic Digital Marketing in the marketing of agricultural products, both in English and Spanish, was analyzed at the time to choose the articles related to the subject the techniques of the aná were used lysis synthesis, inductive and deductive, then
\end{abstract}


make a review of 50 articles alluding to the topic it could be concluded that the existing information in this regard is scarce, the food sector has been very little explored in this area, even in many of the reviewed research still It confuses terms such as Inbound marketing, content marketing and digital marketing, which is why there is much to investigate in this regard, not only as a marketing tool for the agricultural sector, but also in a general way for different economic sectors.

Key words: skills, collaborative culture, leadership, agriculture.

\section{Introducción}

Los principios de la ventaja competitiva cada día son más dependientes de la innovación tecnológica y científica (Andrade, 2016), recibiendo atención considerable tanto a nivel académico como en la práctica (Alves, Barbieux, Reichert, Tello-Gamarra, \& Zawislak, 2017), a fin de alcanzar un buen desempeño que se refleje en el éxito empresarial (Acuna \& Castillo, 2018).

Entre las innovaciones más empleadas en la actualidad se encuentran las tecnologías de la interacción entre cliente y empresa, la cual ya no es controlada por las empresas, es así que, el cliente desempeña dos roles en la actualidad, creadores de valor y al mismo tiempo clientes. Su aporte favorece al cumplimiento de estándares de calidad, los cuales se integran a la ejecución de los procesos (Vite, Romero, \& Vargas, 2018)

La mayoría de países del mundo han asumido posturas de vanguardia en relación al papel de la innovación dentro de la sociedad, y otras regiones del mundo como es el caso de Iberoamérica se encuentran en estado de alerta (Aponte, 2016).

A nivel empresarial, el internet ha provocado cambios significativos, es así como surge la comercialización electrónica, modelo de negociación que cuenta cada día con más seguidores, además de generar grandes beneficios económicos (Perdigón, Viltres, \& Madrigal, 2018). 
Una nueva etapa industrial es la industria 4.0, son varias las tecnologías que emergen a fin de promocionar soluciones digitales, por lo que es importante conocer los patrones de adopción en los diferentes tipos de empresas (Frank, Dalenogare, \& Ayala, 2019). Las cuales deben aportar al desarrollo de los procesos internos en cada organización, con el objeto de tributar el crecimiento organizacional de las empresas (Vite, Palomeque, \& Romero, 2018)

Marketing digital y empresa

Los avances que surgen constantemente en el campo digital están desafiando la comprensión de los mercados y las prácticas de marketing, brindando al demandante una oportunidad emocionante para imaginar las ofertas (Ruyter, Isobel Keeling, \& Ngo, 2018).

La tecnología digital crea interrupciones, las cuales desencadenan respuestas estratégicas de parte de las organizaciones, en busca de alterar sus caminos de creación de valor (Vial, 2019); para ello, la publicidad digital se posiciona en los diferentes espacios pagados de internet como son motores de búsqueda y redes sociales (Aslam \& Karjaluoto, 2017).

Los negocios en internet aprovechan los canales digitales a fin de escalar funciones y servicios, su forma de operar es mediante la conexión y retención de clientes por medio de iniciativas de marketing.

La digitalización de la empresa permite mejorar el mantenimiento preventivo y proactivo, además de ello, también le permite crear y capturar valor por medio de una variedad de componentes software, sin embargo, muchas de estas empresas enfrentan

desafíos tales como: el cliente espera soluciones inteligentes que sean acordes a sus necesidades, a las cuales generalmente son reacios (Gebauer \& Baines, 2019).

Los académicos y mercadólogos han estado intrigados desde la aparición de los medios sociales y la influencia que estos pudieran tener en la disciplina (Salo, 2017). 
La influencia de las redes sociales es cada vez mayor en los negocios, la política y la sociedad, es por ello que, comprender su funcionamiento es muy importante en la actualidad, sus características son similares a las de una epidemia, existe libre interacción (Bhattacharya, Gaurav, \& Ghosh, 2019).

Las redes sociales en línea omnipresentes en la actualidad tienen una influencia cada vez mayor en los negocios, la política y la sociedad. Comprender estos nuevos mecanismos de difusión de información es muy importante para decidir las políticas de campaña. Debido a la libre interacción entre un gran número de miembros, la difusión de información en las redes sociales tiene varias características similares a una epidemia.

La gran cantidad de información generada por el fácil acceso y disponibilidad de las redes sociales, es utilizada por los profesionales del marketing para la obtención de información estratégica y marketing digital

Marketing digital y comercialización de alimentos

En la actualidad, las prácticas tradicionales de marketing no se adaptan a las pequeñas y medianas empresas, esto ha llevado al empresario a desaprender las prácticas tradicionales y reemplazarlas con nuevos e innovadoras acciones (Sadiku, Dana, \& Ramadani, 2019).

Los productos del sector alimentario se comercializan por medio de canales intermedios, sean estas tiendas de comestibles, o la comercialización directa al consumidor, como son los mercados de agricultores, este último, en la actualidad ha alcanzado un nivel máximo (Printezis \& Grebitus, 2018), es por ello que las empresas del sector están en la busca constante de nuevas estrategias para llegar a sus clientes de forma efectiva.

Una de las fuentes más importantes de ventajas competitivas en el marketing es el uso del internet, sea este negocio a negocio o negocio a consumidor (B2C y B2B) (Leeflang, Verhoef, Dahlström, \& Freundt, 2014). 
El uso de Internet sigue explotando en todo el mundo, y lo digital se está convirtiendo en una fuente cada vez más importante de ventajas competitivas en el marketing B2C y B2B. Se ha prestado mucha atención a las enormes oportunidades que presenta el marketing digital, con poca atención a los desafíos reales a los que se enfrentan las empresas a la hora de digitalizarse.

Una de las grandes oportunidades que se están utilizando en la comercialización de bienes y servicios son las redes sociales, las cuales aprovechan el poder de las comunidades, las cuales ofrecen el apalancamiento ideal a los impulsores del marketing empresarial

Las soluciones digitales le han permitido a la bioeconomía dar grandes pasos durante los últimos años, permitiendo visibilizar la cadena de suministro en tiempo real, precisión en la entrega, y optimización del stock acorde con la planificación de la demanda, así como la eliminación de las distancias (Watanabe, Naveed, \& Neittaanmäki, 2018).

En muchos de los casos, la industria de la publicidad es liderada por las empresas comercializadoras de alimentos y bebidas, las cuales han incorporado el marketing interactivo, a fin de acceder principalmente al sector adolescente.

Inbound marketing

El Inbound marketing es la disciplina que se encarga de atraer nuevos clientes de forma natural, su origen busca dar respuesta al segmento de la población cuya forma de búsqueda son las redes sociales, y también a marcas y empresas que requieren de una relación de confianza con los clientes potenciales, la cual no le es posible obtener por medio de los canales tradicionales (Toledo \& San, 2015).

Inbound Marketing hace referencia al proceso de alcanzar y convertir a los consumidores idóneos por medio de la creación y aplicación de tácticas orgánicas en internet, entre sus elementos destacados están la interactividad y el compromiso, los cuales le ofrecen al comercializador la circunstancia adecuada para impulsar 
relaciones valiosas y continuas con los clientes (Opreana \& Vinerean, 2015).

Inbound marketing en la industria alimentaria

En la actualidad, la gran mayoría de productores agrícolas se dirigen a pocos mercados, esto conduce a una inundación de productos, precios bajos y disminución de la calidad de los productos frescos, sin embargo, algunos potenciales mercados siguen sin ser explotados; el uso de la tecnología, permite conectar al agricultor con el mercado, cerrando así la brecha de información existente, al mismo tiempo que puede tomar decisiones en base a información (Masuka et al., 2016).

Entre los grandes desafíos que enfrentan los productores agropecuarios se halla la asimetría de la información, la falta de información de mercado, escaso uso de la telefonía celular como apoyo a la actividad (Samuel, 2018). La tecnología avanza a pasos agigantados, siendo los dispositivos celulares los que concentran las acciones humanas, entre las cuales se encuentran las decisiones de compra.

Los agricultores que utilizan un teléfono móvil como herramienta de búsqueda de información son pocos, la razón principal para ello es la falta de información relevante a la que es posible acceder por medio de la telefonía móvil (Tadesse \& Bahiigwa, 2015).

En el caso de los consumidores, la opción sería la utilización de la tecnología a fin de recibir el producto de origen agrícola directamente en su hogar, sin intermediarios.

Tomar la decisión de compra por parte de los consumidores resulta complejo, lleno de argumentos y conflictos, es por esto que se plantea la necesidad de diseñar servicios de recomendación de productos en base a diferentes criterios a fin de ayudar a los consumidores, entre los cuales se encuentran la ubicación, precio, madurez operacional, madurez del conocimiento y madurez de la estrategia, estos ayudan al consumidor al momento de tomar decisiones de compra (Lian \& Ke, 2016). 
El mejor desempeño empresarial en esta última década, ha sido producto del uso de las Tecnologías de Información y Comunicación (TIC), principalmente en la mercadotecnia (Gutiérrez \& Nava, 2016), sin embargo, esto no ha servido de influencia para que la producción literal relacionada con el tema vaya a la par, principalmente en relación a las pequeñas y medianas empresas, menos aún en las empresas del sector agropecuario, es por esto que se plantea como objetivo un análisis bibliométrico acerca de las tendencias del uso de Inbound marketing en la comercialización de productos agropecuarios.

\section{Materiales y métodos}

La metodología utilizada incluye la búsqueda de información de diferentes artículos científicos importantes, para ello se utilizó las diferentes bases de datos, entre ellas Web of Science y Scopus y Taylor \& Francis, los términos que se utilizaron fueron "Marketing digital e Inbound marketing", además de ello, se analizó el tema específico Marketing digital en la comercialización de productos agrícolas.

Existe una gran cantidad de trabajos realizados en relación al marketing digital, sin embargo, la elección del contenido deberá adaptarse al objetivo propuesto en la investigación, el uso del marketing digital y el Inbound marketing en los agricultores, las publicaciones científicas a las que se acceda deberán ser adecuadas para el contexto agrícola.

A fin de hacer una buena revisión bibliográfica, al momento de escoger los artículos relacionados con la temática se utilizaron las técnicas del análisis síntesis, inductivo y deductivo, los cuales correspondieron a los años 2016, 2017, 2018 y 2019, tanto en inglés como en español.

\section{Resultados}

Estudios realizados con la utilización de Inbound marketing en la comercialización de productos agrícolas 
En el contexto actual, la competencia entre empresas por atraer demandantes de los productos comercializados ha llevado a la implementación de nuevas y diferentes estrategias de mercadeo, entre las estrategias utilizadas en cierta medida se encuentra el Inbound marketing, marketing entrante o interactivo (Royo \& Hünermund, 2016).

El marketing digital involucra crear una relación de confianza y lealtad con el cliente, es por ello que su principal aspecto es el Inbound marketing o marketing entrante (Baltes, 2016). En este escenario se establece en la tabla siguiente el detalle de las revisiones realizadas.

\section{Tabla 1}

Resultado de las investigaciones realizadas

\begin{tabular}{|c|c|c|}
\hline Método utilizado & Sistema & Autor \\
\hline $\begin{array}{l}\text { MCDM } \\
\text { (ELECTRE } \\
\text { modificado) }\end{array}$ & $\begin{array}{l}\text { Sistema de } \\
\text { recomendación } \\
\text { móvil }\end{array}$ & (Lian \& Ke, 2016). \\
\hline $\begin{array}{l}\text { Soluciones } \\
\text { virtuales } \\
\text { aumentadas }\end{array}$ & $\begin{array}{l}\text { Marketing } \\
\text { sensorial }\end{array}$ & $\begin{array}{l}\text { (Petit, Velasco, \& Spence, } \\
\text { 2019). }\end{array}$ \\
\hline Big Data & $\begin{array}{l}\text { Marketing } \\
\text { multisensorial }\end{array}$ & $\begin{array}{l}\text { (Yoganathan, Osburg, \& } \\
\text { Akhtar, 2019) }\end{array}$ \\
\hline Big Data & Tecnología y salud & $\begin{array}{l}\text { (K. Montgomery, Chester, } \\
\text { Nixon, Levy, \& Dorfman, } \\
\text { 2019) }\end{array}$ \\
\hline $\begin{array}{l}\text { Zomato } \\
\text { (software) }\end{array}$ & $\begin{array}{l}\text { Entrega de } \\
\text { alimentos en línea }\end{array}$ & (Raman, 2018) \\
\hline $\begin{array}{l}\text { Parlante } \\
\text { inteligente }\end{array}$ & $\begin{array}{l}\text { Dispositivo } \\
\text { inalámbrico } \\
\text { dotado } \\
\text { inteligencia } \\
\text { artificial }\end{array}$ & (Smith, 2018) \\
\hline Redes sociales & $\begin{array}{lr}\begin{array}{l}\text { Interacción } \\
\text { clientes }\end{array} & \begin{array}{r}\text { con } \\
\text { en }\end{array} \\
\text { Facebook } & \\
\end{array}$ & $\begin{array}{l}\text { (de Vries, Veer, \& de Vries, } \\
\text { 2018) }\end{array}$ \\
\hline Encuesta & $\begin{array}{l}\text { Inbound marketing } \\
\text { y comerciantes } \\
\text { detallistas }\end{array}$ & (Terán et al., 2018) \\
\hline Encuesta & Hamburgueserías & (Cárdenas, 2018) \\
\hline
\end{tabular}




\begin{tabular}{|c|c|c|}
\hline $\begin{array}{l}\text { Marketing } \\
\text { realidad } \\
\text { aumentada }\end{array}$ & $\begin{array}{l}\text { Aplicaciones } \\
\text { móviles de AR }\end{array}$ & $\begin{array}{l}\text { (Rauschnabel, } \\
\text { Hinsch, 2019) }\end{array}$ \\
\hline $\begin{array}{l}\text { Marketing de } \\
\text { realidad } \\
\text { aumentada }\end{array}$ & Compra móvil & (Scholz \& Duffy, 2018) \\
\hline $\begin{array}{l}\text { Marketing } \\
\text { contenidos }\end{array}$ & $\begin{array}{l}\text { Agroproductos } \\
\text { derivados del } \\
\text { sector } \\
\text { agropecuario }\end{array}$ & $\begin{array}{l}\text { (Vite, Sánchez, \& Freire, } \\
\text { 2018) }\end{array}$ \\
\hline $\begin{array}{l}\text { Wallapop, } \\
\text { Westwing } \\
\text { Fotocasa }\end{array}$ & Compra móvil & (Coll, 2019) \\
\hline Growth hacking & $\begin{array}{l}\text { Captación } \\
\text { audiencias }\end{array}$ & (Coll \& Micó, 2019) \\
\hline Marketing Digital & $\begin{array}{l}\text { Mypimes } \\
\text { alimentos }\end{array}$ & (Armijos Delgado, 2019) \\
\hline Realidad virtual & $\begin{array}{l}\text { Enfoque de } \\
\text { minería de texto }\end{array}$ & $\begin{array}{l}\text { (Maria, Loureiro, Guerreiro, } \\
\text { Eloy, \& Langaro, 2018) }\end{array}$ \\
\hline $\begin{array}{l}\text { Servitización } \\
\text { digital }\end{array}$ & $\begin{array}{lr}\text { Análisis } & \text { del } \\
\text { ecosistema } & \text { de } \\
\text { servicios } & \end{array}$ & $\begin{array}{l}\text { Sklyar, Kowalkowski, } \\
\text { Tronvoll, \& Sörhammar, } \\
\text { 2019) }\end{array}$ \\
\hline $\begin{array}{l}\text { Marketing digital } \\
\text { en la viticultura }\end{array}$ & $\begin{array}{l}\text { Publicidad en la } \\
\text { viticultura }\end{array}$ & $\begin{array}{l}\text { (Denić, Petković, Siljković, } \\
\text { \& Ivković, 2019) }\end{array}$ \\
\hline $\begin{array}{l}\text { Realidad } \\
\text { aumentada }\end{array}$ & $\begin{array}{l}\text { Realidad } \\
\text { aumentada en el } \\
\text { entorno minorista }\end{array}$ & (van Esch et al., 2019) \\
\hline $\begin{array}{l}\text { Catálogos } \\
\text { digitales } \\
\text { enriquecidos }\end{array}$ & Caso Ikea & (Garnier \& Poncin, 2019) \\
\hline
\end{tabular}

\section{El Autor}

\section{Investigaciones realizadas en Inbound Marketing}

Si bien existe mucha literatura científica relevante, el Inbound Marketing es un tema muy poco investigado, más aún en el sector alimentario y propiamente enfocado en la comercialización de productos agropecuarios, la mayoría de ellos tienen un enfoque en alimentos procesados, los cuales si bien es cierto se clasifican como alimentos, sin embargo, muchos de ellos ya no son $100 \%$ naturales como el caso de bebidas, hamburguesas, etc. 
En América latina es muy poca la información para el sector alimentario, la mayor cantidad de información se obtuvo de países desarrollados como España y Estados Unidos, en los cuales existen incluso aplicaciones móviles para su distribución y venta.

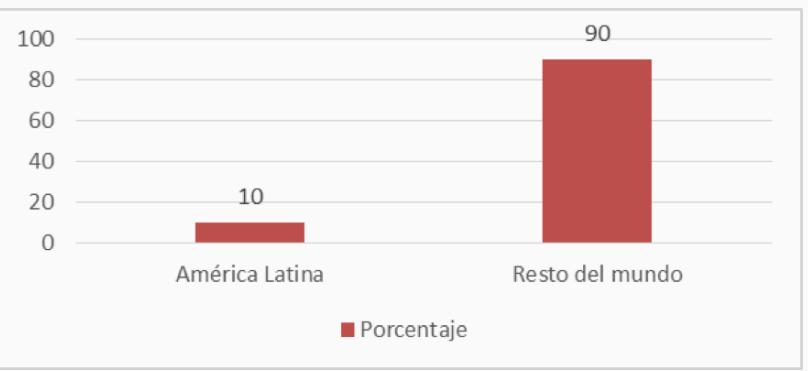

\section{Gráfico 1}

Investigaciones realizadas de Inbound Marketing en el sector alimentario

\section{Evolución temporal de la productividad}

Para obtener la evolución temporal de la productividad por año de publicación, se procedió a obtener la frecuencia de publicaciones realizadas por año, análisis que reflejo un incremento de publicaciones referentes al tema durante los años 2018 y 2019, siendo el año de menor productividad el 2017.

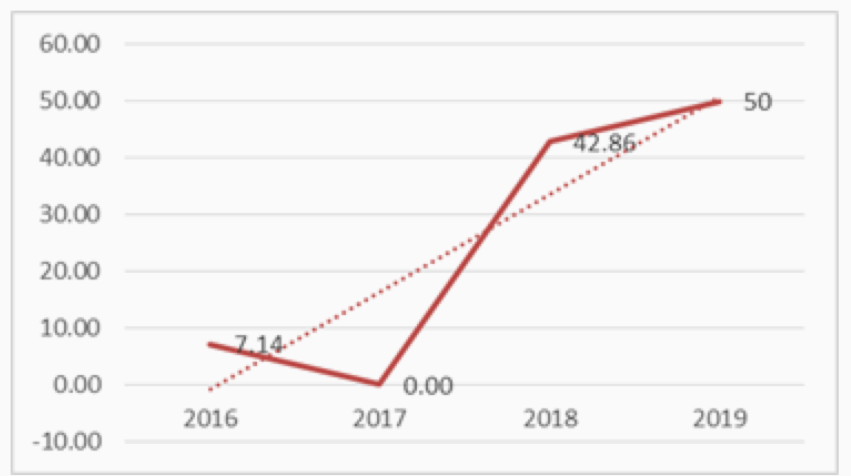

\section{Gráfico 2}

Productividad científica

\section{Productividad científica por región}

Las publicaciones científicas investigadas procedieron de diferentes partes del mundo, sin embargo, solo el 14,29\% han sido realizados 
en Ecuador y en América Latina, el $71,43 \%$ restante han sido realizadas en otros países del mundo.

\begin{tabular}{|l|l|l|l|}
\hline 80.00 & & \\
70.00 & & \\
60.00 & & \\
50.00 & & \\
40.00 & & & \\
30.00 & & & \\
20.00 & & & \\
10.00 & 14.29 & Mundo \\
\hline 0.00 & América Latina & Ecuador & Mudor \\
\hline
\end{tabular}

Gráfico 3 Productividad científica por región

\section{Marketing sensorial}

Una oportunidad única para el sector alimentario puede ser el marketing sensorial, la población consume y compra cada vez más productos en línea, medio en el cual la interacción sensorial ha estado limitada solo a actividades visuales y auditivas, es por ello que los mercadólogos e investigadores deben contar con una visión de futuro y empoderarse de las ultimas herramientas tecnológicas a fin de ofrecer experiencias más ricas al consumidor (Petit, Velasco, \& Spence, 2019).

La próxima frontera del marketing sensorial es sin duda el entorno en línea, las técnicas multisensoriales brindan un futuro prometedor (Yoganathan, Osburg, \& Akhtar, 2019).

Una forma esencial de acercarse a los clientes en la actualidad son las redes sociales, mediante el empleo de ellas las empresas no solo venden, también es posible conocer opiniones y comentarios de sus clientes, así como sus preferencias (Raman, 2018).

\section{Discusión}

En la actualidad existe una verdadera batalla por la visibilidad y diferenciación, el sector de alimentos no es la excepción, por medio del marketing digital y el Inbound marketing, el problema está resuelto, incluso se genera un sistema de mensajería que aporta valor 
con respecto a la competencia, además de ello, cada día se crean nuevas oportunidades de promocionar los productos por este medio, entre ellos el marketing sensorial y la entrega de alimentos en línea.

Otros aspectos que se debe tener en cuenta solo las publicaciones científicas fueron obtenidas en los países desarrollados en comparación a América latina, que a pasar del tempo pudieron aprovechar los avances tecnológicos, desarrollando nuevas formas de realizar marketing mediantes distintos medios como las aplicaciones móviles o redes sociales.

\section{Conclusiones}

Si bien existe información relevante al respecto, el sector alimentario ha sido muy poco explorado, a pesar de tratarse de un sector de mucha importancia, más aun, mucha de la literatura todavía confunde términos como Inbound marketing, marketing de contenidos y marketing digital, razón por la cual hay mucho que investigar al respecto, no solo como herramienta de comercialización para el sector agropecuario, sino también de manera general para los diferentes sectores económicos.

\section{Referencias}

Acuna, C. y Castillo, M. (2018). Barriers to non-technological innovation: Impact on business performance in an emerging economy. Contaduría y Administración, 63(3), 1-23. doi: 10.22201/fca.24488410e.2018.1383

Alves, A. C., Barbieux, D., Reichert, F. M., Tello-Gamarra, J. y Zawislak, P. A. (2017). Innovation and Dynamic Capabilities of the Firm: Defining an Assessment Model. Revista de Administração de Empresas, 57(3), 232-244. doi: 10.1590/s0034-759020170304

Andrade, D. (2016). Revista Escuela de Administración de Negocios. Revista Escuela de Administración de Negocios, 1(80), 59-72.

Recuperado

de https://journal.universidadean.edu.co/index.php/Revista/article/vie $\mathrm{w} / 1457 / 1410$

Aponte, G. M. (2016). Gestión de la innovación tecnológica mediante el análisis de la información de patentes. Negotium, 11(33), 
Armijos Delgado, N. M. (2019). Marketing Digital: una herramienta para potenciar y promocionar las Mypimes en el ámbito internacional. INNOVA Research Journal, 4(1), 1-8. doi: 10.33890/innova.v4.n1.2019.764

Aslam, B. y Karjaluoto, H. (2017). Digital advertising around paid spaces, E-advertising industry's revenue engine: A review and research agenda. Telematics and Informatics, 34(1), 1650-1662. doi: 10.1016/j.tele.2017.07.011

Baltes, L. P. (2016). Inbound marketing - The most important digital marketing strategy. Bulletin of the Transilvania University of Braşov. Series V: Economic Sciences, 9(2), 61-68. Recuperado de http://webbut.unitbv.ro/BU2016/Series\%20V/BULETIN\%20I/07_ Patrutiu_Baltes.pdf

Bhattacharya, S., Gaurav, K., y Ghosh, S. (2019). Viral marketing on social networks: An epidemiological perspective. Physica A: Statistical Mechanics and Its Applications, 525(1), 478-490. doi:10.1016/j.physa.2019.03.008

Cairns, G. (2013). Evolutions in food marketing, quantifying the impact, and policy implications. Appetite, 62(1), 194-197. doi:10.1016/j.appet.2012.07.016

Cárdenas, A. (2018). Inbound marketing para las hamburgueserías del municipio San Francisco. Marketing Visionario, 7(1), 42-56. Recuperado de http://ojs.urbe.edu/index.php/market/article/view/1714/2592

Coll, Patricia, y Micó, J. L. (2019). Growth hacking in the strategy to attract audiences in the new economy. Hipertext.Net: Revista Académica Sobre Documentación Digital y Comunicación Interactiva, 1(18), 14-22. doi: 10.31009/hipertext.net.2019.i18.02

Coll, Patricio. (2019). El marketing de contenidos en la estrategia de growth hacking en la nueva economía. Los casos de Wallapop, Westwing y Fotocasa. AdComunica. Revista Científica de Estrategias, Tendencias e Innovación En Comunicación, 1(17), 105116. doi: 10.6035/2174-0992.2019.17.7 
De Vries, H. P., Veer, E. y de Vries, K. V. (2018). An examination of SME social media use in the food industry. Small Enterprise Research, 25(3), 227-238. doi: 10.1080/13215906.2018.1521741

Denić, N., Petković, D., Siljković, B. y Ivković, R. (2019). Opportunities for Digital Marketing in the Viticulture of Kosovo and Metohija. Reference Module in Materials Science and Materials Engineering, 1(1)1-16. doi: 10.1016/b978-0-12-803581-8.11592-9

Frank, A. G., Dalenogare, L. S. y Ayala, N. F. (2019). Industry 4.0 technologies: Implementation patterns in manufacturing companies. International Journal of Production Economics, 210(1), 15-26. doi: 10.1016/j.ijpe.2019.01.004

Garnier, M. y Poncin, I. (2019). Do enriched digital catalogues offer compelling experiences, beyond websites? A comparative analysis through the IKEA case. Journal of Retailing and Consumer Services, 47(1), 361-369. doi: 10.1016/j.jretconser.2018.12.011

Gebauer, H. y Baines, T. (2019). Digital servitization business models in ecosystems: A theory of the firm. Journal of Business Research, 104(1), 380-392. doi: 10.1016/j.jbusres.2019.06.027

Gutiérrez, C. y Nava, R. (2016). Mercadotecnia digital y las pequeñas y medianas empresas: revisión de la literatura. Enl@ce: Revista Venezolana de Información, Tecnología y Conocimiento, 13(1), 45-61. Recuperado de http://www.redalyc.org/pdf/823/82346016004.pdf

Leeflang, P. S. H., Verhoef, P. C., Dahlström, P. y Freundt, T. (2014). Challenges and solutions for marketing in a digital era. European Management Journal, 32(1), 1-12. doi: 10.1016/j.emj.2013.12.001

Lian, J. W. y Ke, C. K. (2016). Using a modified ELECTRE method for an agricultural product recommendation service on a mobile device. Computers and Electrical Engineering, 56(1), 277-288. doi: 10.1016/j.compeleceng.2015.11.014

Maria, S., Loureiro, C., Guerreiro, J., Eloy, S. y Langaro, D. (2018). Understanding the use of Virtual Reality in Marketing: A text mining-based review. Journal of Business Research, 100(1), 514530. doi: 10.1016/j.jbusres.2018.10.055 
Masuka, B., Matenda, T., Chipomho, J., Mapope, N., Mupeti, S., Tatsvarei, S. y Ngezimana, W. (2016). Mobile phone use by smallscale farmers: a potential to transform production and marketing in Zimbabwe. South African Journal of Agricultural Extension (SAJAE), 44(2), 121-135. doi: 10.17159/24133221/2016/v44n2a406

Montgomery, K. C. y Chester, J. (2009). Interactive Food and Beverage Marketing: Targeting Adolescents in the Digital Age. Journal of Adolescent Health, 45(3), S18-S29. doi: 10.1016/j.jadohealth.2009.04.006

Montgomery, K., Chester, J., Nixon, L., Levy, L. y Dorfman, L. (2019). Big Data and the transformation of food and beverage marketing: undermining efforts to reduce obesity? Critical Public Health, 29(1), 110-117. doi: 10.1080/09581596.2017.1392483

Opreana, A. y Vinerean, S. (2015). A new development in online marketing: Introducing digital inbound Marketing. Expert Journal of Marketing, 3(1), 29-34. Recuperado de http://marketing.expertjournals.com/23446773-305/

Perdigón, R., Viltres, H. y Madrigal, I. (2018). Estrategias de comercio electrónico y marketing digital para pequeñas y medianas empresas. Revista Cubana de Ciencias Informáticas, 12(3), 17. Recuperado de http://rcci.uci.cu

Petit, O., Velasco, C. y Spence, C. (2019). Digital Sensory Marketing: Integrating New Technologies Into Multisensory Online Experience. Journal of Interactive Marketing, 45(1), 42-61. doi: 10.1016/j.intmar.2018.07.004

Printezis, I. y Grebitus, C. (2018). Marketing Channels for Local Food. Ecological Economics, 152(1), 161-171. doi: 10.1016/j.ecolecon.2018.05.021

Raman, P. (2018). Zomato: a shining armour in the foodtech sector. Journal of Information Technology Case and Application Research, 20(3-4), 130-150. doi: 10.1080/15228053.2018.1552396

Rauschnabel, P., Felix, R. y Hinsch, C. (2019). Augmented reality marketing: How mobile AR-apps can improve brands through inspiration. Journal of Retailing and Consumer Services, 49(1), 4353. doi: 10.1016/j.jretconser.2019.03.004 
Royo, M. y Hünermund, U. (2016). Effects of inbound marketing communications on HEIs' brand equity: the mediating role of the student's decision-making process. An exploratory research. Journal of Marketing for Higher Education, 26(2), 143-167. doi: 10.1080/08841241.2016.1233165

Ruyter, K. de, Isobel Keeling, D. y Ngo, L. V. (2018). When nothing is what it seems: A digital marketing research agenda. Australasian Marketing Journal, 26(3), 199-203. doi: 10.1016/j.ausmj.2018.07.003

Sadiku, N., Dana, L. y Ramadani, V. (2019). Entrepreneurial marketing dimensions and SMEs performance. Journal of Business Research, 100(1), 86-99. doi: 10.1016/j.jbusres.2019.03.025

Salo, J. (2017). Social media research in the industrial marketing field: Review of literature and future research directions. Industrial Marketing Management, 66(1), 115-129. doi: 10.1016/j.indmarman.2017.07.013

Samuel, M. (2018). Mobile Phone Use by Zimbabwean Smallholder Farmers: A Baseline Study. The African Journal of Information and Communication, 22(1), 29-52. doi: 10.23962/10539/26171

Scholz, J. y Duffy, K. (2018). We ARe at home: How augmented reality reshapes mobile marketing and consumer-brand relationships. Journal of Retailing and Consumer Services, 44(1), 11-23. doi: 10.1016/j.jretconser.2018.05.004

Sklyar, A., Kowalkowski, C., Tronvoll, B. y Sörhammar, D. (2019). Organizing for digital servitization: A service ecosystem perspective. Journal of Business Research, 104(1), 450-460. doi: 10.1016/j.jbusres.2019.02.012

Smith, K. T. (2018). Marketing via smart speakers: what should Alexa say? . Journal of Strategic Marketing,1(1) 1-16. doi: 10.1080/0965254X.2018.1541924

Tadesse, G. y Bahiigwa, G. (2015). Mobile Phones and Farmers' Marketing Decisions in Ethiopia. World Development, 68(1), 296307. doi: 10.1016/j.worlddev.2014.12.010

Terán, O., Espinoza, E., Márquez, O., Hernández, P., Ruíz, E., Vergara, M. y Lara, M. (2018). Inbound marketing como estrategia competitiva para el comercio detallista de las mipymes en el 
municipio de Juchitepec, estado de México. Revista Global de Negocios, 6(7), 87-97. Recuperado de http://www.theibfr2.com/RePEc/ibf/rgnego/rgn-v6n7-2018/RGNV6N7-2018-8.pdf

Toledo, F. y San, B. (2015). Herramientas de marketing de contenido para la generacion de tráfico cualificado online. Opción, 31(4), 978996.

Van Esch, P., Arli, D., Gheshlaghi, M., Andonopoulos, V., von der Heidt, T. y Northey, G. (2019). Anthropomorphism and augmented reality in the retail environment. Journal of Retailing and Consumer Services, 49(1), 35-42. doi: 10.1016/j.jretconser.2019.03.002

Vial, G. (2019). Understanding digital transformation: A review and a research agenda. Journal of Strategic Information Systems, 28(2), 118-144. doi: 10.1016/j.jsis.2019.01.003

Vite, H., Palomeque, I., \& Romero, W. (2018). Análisis de calidad del servicio de atención al afiliado en Centros de Salud del IESS: Caso Santa Rosa y Pasaje. Revista de Investigación Talentos, 5(2), 68-78.

http://talentos.ueb.edu.ec/index.php/talentos/article/view/44

Vite, H., Romero, W., \& Vargas, J. (2018). Análisis de procesos en unidades de atención al usuario en instituciones públicas caso de estudio: dirección provincial IESS el Oro. SATHIRI: Sembrador, 13(1), 170-182. Recuperado de http://revistasdigitales.upec.edu.ec/index.php/sathiri/article/view/51 0

Vite, H., Sánchez, W. y Freire, F. (2018). AGROPRODUCTOSEC DERIVADOS DEL SECTOR AGROPECUARIO APLICANDO. Revista Observatorio de La Economía Latinoamericana, 1(1), 1-16. Recuperado de https://www.eumed.net/rev/oel/2018/10/comercializacionagroproductosec.html

Watanabe, C., Naveed, N. y Neittaanmäki, P. (2018). Digital solutions transform the forest-based bioeconomy into a digital platform industry - A suggestion for a disruptive business model in the digital economy. Technology in Society, 54(1), 168-188. doi: 1016/j.techsoc.2018.05.002 
Yoganathan, V., Osburg, V. S. y Akhtar, P. (2019). Sensory stimulation for sensible consumption: Multisensory marketing for etailing of ethical brands. Journal of Business Research, 96(1), 386396. doi: 10.1016/j.jbusres.2018.06.005 